Valentina Perrone

\title{
BREVI RIFLESSIONI SULL'ORIGINE DELL'ECCEZIONE GENERALE DI DOLO
}

\section{Premessa}

La complessità del traffico giuridico contemporaneo, propone il superamento della dimensione statuale e rende consapevoli dell'inadeguatezza delle normative (soprattutto delle leggi) rispetto alla poliedricità di situazioni della realtà economica e giuridica dell'oggi.

Ne conseguono la crisi delle fonti normative (a partire dalla inattualità dei 'Co-

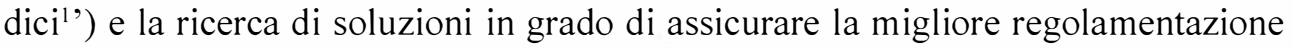
giuridica rispetto al caso concreto e riguardo alla singola persona, con la tendenza ad attivare processi di unificazione delle regole giuridiche connesse ai traffici commerciali, mediante molteplici tecniche. Di volta in volta, nei differenti tentativi esperiti, si è perseguita la via del cosiddetto "diritto uniforme" o quella dell'individuazione di un generale lex mercatoria ${ }^{2}$ nata dall'agglomerato e dalla stratificazione di con-

R. David e C. Jauffret-Spinosi, I grandi sistemi giuridici contemporanei, [cur. R. Sacco], Padova, 2004, p. 92 ss.: «Fra le leggi ordinarie alcune ricevono il nome di codici. La parola era impiegata, all'origine, per indicare talune raccolte in cui erano riunite diverse leggi: tali sono il codice teodosiano e il codice di Giustiniano. Nel XIX secolo è parso che la denominazione si dovesse riservare a certe compilazioni che miravano ad esporre i principi di uno ius commune moderno, dichiarato applicabile in uno Stato, ma che si vorrebbe applicare in un modo universale, in opposizione alle regole, ispirate da considerazioni di opportunità più che di giustizia, che continuerebbero ad esistere nell'ambito di ogni nazione. Tuttavia la terminologia non è ben stabilita e attualmente la parola codice è largamente utilizzata per indicare compilazioni che tendono a raggruppare e a esporre sistematicamente la regolamentazione relativa ad una materia particlare. In una o nell'altra forma, il movimento di codificazione si è esteso, nel XIX e nel XX secolo, a tutti i paesi della famiglia romano-germanica. La parentela tra gli ordinamenti di questi paesi si è manifestata non soltanto in questa adesione comune alla formula della codificazione, ma anche nel modo con cui le regole sono state coordiante in un certo numero di codici. La Francia aveva fornito un esempio, promulgando i cinque codici napoleonici. Nei diversi paesi della famiglia romano-germanica si ritrvano gli stessi cinque codici fndamentali. In Europa, la sola eccezzione da notare è rappresentata dai paesi nordici. Un codice unico è stato promulgato in ognuno di questi paesi: nel 1683 in Danimarca e nel 1687 in Norvegia, nel 1734 per la Svezia e la Finlandia. Questi codici, molto anteriori alla codificazione napoleonica, comprendevano l'insieme del diritto, come hanno fatto in seguito in Prussia l'Allgemeines Landrecht (1794) e in Russia I Svod Zakonov (1832). Un'evoluzione divergente è avvenuta in seguito nei due paesi nordici. I codici hanno cessato in pratica di esistere in Danimarca e in Norvegia, così come in Islanda; in questi paesi, le loro diverse parti sono state abrogate e sostituite con grandi leggi, non integrate nei vecchi codici.

2 F. Galgano, La globalizzazione nellospecchio del diritto, Bologna, 2005, p. 56 s.: «Si va ricomponendo, nel tempo presente, l'antico rapporto tra il diritto e l'economia. Al giurista odierno non si chiede soltanto di interpreta- 
suetudini, spesso secolari; con lo stesso intento si è dato vita a progetti di nuovi Codici.

Tutto ciò allo scopo di meglio tutelare le esigenze e le aspettative dell'uomo, per le quali occorrono rimedi che abbiano sufficiente elasticità e siano in grado di far corrispondere la disciplina giuridica alla realtà fattuale.

Ciò ha prodotto sia interrogativi di tipo sostanziale concernenti la concreta norma da applicare alla singola fattispecie, sia di diritto processuale poiché sono sorte anche nuove Corti adibite all'esame ed alla tutela della persona.

Nello sforzo ermeneutico che ne è conseguito ha avuto risalto il ricorso a princípi e criteri nuovi ed in grado di andare di là dalla disciplina specifica prevista nelle pattuizioni e nelle leggi.

Tra essi ha assunto un ruolo di crescente importanza il richiamo alla buona fede oggettiva $^{3}$; la quale, come è pacificamente affermato sia sul piano della logica del sistema e delle categorie che la compongono sia circa la concreta applicabilità propone uno schema operativo diverso dalla più nota e radicata tutela della buona fede in senso soggettivo.

Il punto è stato approfondito dalla dottrina recenziore, la quale unanimemente ha colto le profonde differenze esistenti tra le due categorie, della buona fede sog-

re la legge; gli si chiede come nell'epoca preindustriale, di utilizzare la tecnica giuridica per soddisfare i sempre mutevoli bisogni del mercato dando forma a nuovi beni o a nuove operazioni economiche; e la formula law and economics, coniata negli Stati Uniti per designare un'area disciplinare (oltre che un'apposita enciclopedia) e da qualche tempo ripresa anche in Europa, attesa la ricostruita continuità fra il diritto e leconomia. Al tempo stesso la società globale si da un proprio diritto nn statuale, al quale si è attribuito il nome di nuova lex mercatria. L'espressione ha origine colta: vuole alludere alla rinascita, in epoca moderna, di un diritto altrettanto universale quant fu universale il diritto dei mercanti dell'epoca intermedia. Questo era stato lex mercatoria, non solo perché regolava i rapporti mercantili, ma anche e soprattutto perché era un diritto creato dai mercanti: le sue fonti erano state gli statuti delle corporazioni mercantili, le consuetudini mercantili, la giurisprudenza delle curiae mercatorum, che eran tali a loro volta perché in esse sedevano mercanti. Del pari, per la nuova lex mercatoria oggi si intende un diritto creato dal ceto imprenditoriale, e formato da regole destinate a disciplinare in modo uniforme, al di là delle unità politiche degli Stati, i rapporti commerciali che si instaurano entro l'unità economica dei mercati. L'antica lex mercatoria aveva preceduto l'avvento degli Stati moderni; la sua funzione era consistita nel derogare, per i rapporti commerciali, al diritto civile di allora, ossia al diritto romano, rivelatosi non più congeniale alle esigenze dei traffici. La nuova lex mercatoria opera, per contro, entro una realtà caratterizzata dalla divisione politica dei mercati in una pluralità di Stati; la sua funzione è di superare la discontinuità giuridica da questi provocata. Entro questa nuova lex mercatoria si dissolvono tanto i particolarismi giuridici delle codificazini quanto, fenomeno ancora più significativo, le differenze fra civil law e il common law». L'a. continua affermando (p. 60): «Ha cosi preso vita un diritto uniforme spontaneo; e della lex mercatoria si parla in giurisprudenza come di 'un ordinamento giuridico', separato dagli ordinamenti statuali e dotato, al pari di questi, del carattere di ordinamento originario, quale espressione della business community o societas mercantile».

3 La materia ha generato una copiosissima letteratura ed interessanti filoni giurisprudenziali, dei quali non ritengo di dover far menzione in questa sede, riservandomi di richiamarli nel prosieguo dell'esposizione, in relazione a punti specifici per i quali si è ritenuto opportuno o/e necessario far ricorso alla buona fede oggettiva. Qui rinvio alle rispettive voci delle raccolte enciclopediche: S. Romano, Buona fede (dir. priv.), in Enc. dir., V, Milano, 1959, 684; A. Montel, voce Buona fede, in Nuovis. dig., II, Torino, 1958, 502; R. Nicolò, Diritto Civile, (ad vocem), Enc. Dir., XII, Milano, 1964; L. Bigliazzi Geri, Buona fede nel diritto civile, in Dig. Disc. Priv. Sez. civ., Torino, 1988, II, 176 ss. 
gettiva e della buona fede oggettiva, pur non nascondendosi la difficoltà di delineare con precisione i contorni della buona fede oggettiva ${ }^{4}$.

\section{Dunque, mentre la buona fede soggettiva, può essere intesa come «lo sta-} to psicologico di un soggetto che agisce o si trova in una determinata situazione nell'ignoranza di ledere - o di aver leso - con il suo comportamento, o con il trovarsi o l'essersi trovato in una determinata posizione, un interesse altrui giuridicamente

In proposito, tra i tanti, vorrei menzionare le acute osservazioni svolte, in occasione del Convegno Internazionale sulla buona fede oggettiva in onore di Alberto Burdese, dal Talamanca, profondo conoscitore delle radici dei concetti di buona fede: M. Talamanca, La bona fides nei giuristi romani, in II ruolo della buona fede oggettiva nell'esperienza giuridica storica e contemporanea, Atti del Convegno internazionale di studi in onore di Alberto Burdese, [cur. L. Garofalo], vol. IV, Padova, 2003, pp. 1 ss. L'a. approfondisce la differenza tra buona fede soggettiva e buona fede oggettiva, in relazione alle fonti storiche e al loro riverbero nelle nozioni recepite dal Codice Civile italiano (p. 7-13), offrendo un quadro incisivo, che mi pare possa essere assunto come un valido punto di riferimento per la presente analisi: «Fra le due categorie è, senz'altro, molto più difficile, non dico da definire, bensi anche da descrivere la buona fede oggettiva rispetto a quella soggettiva. In un sistema codicistico, quale è il nostro attuale, il primo punto di riferimento per una discussione sulle categorie da impiegare è rappresentato dall'uso dei termini, e dai correlati schemi concettuali, fatto dal legislatore, anzitutto - meglio esclusivamente - nel codice. In effetti, a fronte dell'impiego senz'altro ampio, della categoria della buona fede da intendersi in senso soggettivo, la buona fede nella sua valenza oggettiva è considerata in non molti articoli del codice, fra i quali va riportato, al di là dei possibili dubbi, anche un articolo che, letteralmente, ad essa non si riferisce. E', d'altronde, è la sola buona fede in senso soggettivo che - in tema di possesso - il legislatore italiano si è ritenuto in grado di definire indirettamente nell'art. 1147 co. I, c.C., nonché per un caso particolare, nell'art. 535, co. III, c.c. La buona fede oggettiva non viene mai definita negli articoli in cui ad essa si fa ricorso: e, anzi, la disciplina dei relativi aspetti può creare problemi per la ricostruzione della categoria e di quelle eventualmente concorrenti. Va fatta una premessa, che potrebbe apparire attualmente del tutto superflua, dato che la distinzione tra buona fede soggettiva e buona fede oggettiva non appare posta in dubbio, anzi di essa e della - astrattamente possibile - costruzione di una categoria generale della buona fede non si prende neppure contezza. Non mi sembra attualmente discutibile, né sul piano della logica del sistema e delle categorie che lo compongono né dal punto di vista della concreta operatività, che la buona fede soggettiva e quella oggettiva siano due schemi operativi diversi. La prima facilmente caratterizzabile sulla base delle definizioni legislative già viste, è lo stato psicologico di un soggetto che agisce o si trova in una detrminata situazione nell'ignoranza di ledere - o di aver leso - con il suo comportamento, o con il trovarsi o l'essersi trovato in una detrminata posizione, un interesse altrui giuridicamente protetto e, in linea di massima, poziore a quello del soggetto in questione. E' tale stato psicologico a rendere tutelabile l'interesse del soggetto in buona fede, a cui viene eventualmente subordinato l'interesse che egli ignora di ledere: ed è questa buona fede in senso soggettivo che si contrappone, in negativo, la mala fede. La buona fede soggettiva - come, del resto, la categoria di segno inverso, la mala fede, che è solo soggettiva nella terminlogia del codice - è, dunque, essenzialmente uno stato psicologico del soggetto, in relazione alla cui sfera di interessi si debbono o non si debbono produrre determinati effetti. Da qualsiasi valutazione di un tale stato prescinde invece la buona fede oggettiva, di cui sul piano della rilevanza come schema operativo e concettuale - manca nel nostro codice una precisa definizione. Al di là, infatti, del generico richiamo, di un'operatività della buona fede il riferimento più concreto è quello dell'art. 1175 c.c dove-sembrerebbe una sorta di paradosso - non v'è un esplicito richiamo allo schema in questione, ma si sancisce che entrambe le parti del rapporto obbligatrio debbono comportarsi secondo le regole della correttezza. A me sembra trattarsi di una norma a carattere generale, di cui sono disposizioni applicative ad un certo limite non necessarie - soprattutto ed in una dottrina e in una giurisprudenza fortemente orientate in senso creativo - le norme che si richiamano all'operatività della buona fede in senso oggettivo, soprattutto quando, come accade nell'art. 1337 e nell'art. 1358 c.c., si faccia riferimento ad un dovere di comportarsi 'secondo buona fede': e non diversamente si deve giudicare la prescrizione che il contratto - vale a dire le obbligazioni da esso nascenti - debba essere eseguito 'secondo buona fede' che si trova nell'art. 1375 c.c. In definitiva, mantenendosi sul piano del nostro codice, la buona fede impone alle parti di un rapporto bbligatorio di comportarsi come un uomo perbene, un galantuomo, il bonus et diligens paterfamilias della tradizione romanistica, trasportato dalla tematica della colpa a quella delle regle di correttezza. Essa si risolve, così, in uno strumento che, se non indica in sé precisi modelli di cmportamento, ne veicla però la rilevanza sul piano della valutazione dei casi concreti, e può - ma in via soltanto metonimica - essere adoperato come una designazione collettiva per tali modelli». Il punto è scandagliato, tra i tanti, da: P. Perlingieri, Manuale di diritto civile, Napoli, 2007, 213; U. Natoli, L'attuazione del rapporto obbligatorio, in Trattato Cicu-Messineo, Milano, 1969, 132 ss.; F. Gazzoni, Manuale di diritto privato, Napoli, 2000, 548 ss.; A.G. Di Majo, Delle obbligazioni in generale, in Comm. Cod. Civ., Scialoja Branca, Bologna-Roma, 1998, sub art. 1175, (part. a), 302. Ritengo doveroso rammentare anche: G. Panza, Prescrizione, in Dig. Disc. Priv., Sezione Civile, XIV, Torino 1997, 226. 
protetto e, in linea di massima, poziore a quello del soggetto in questione $\rangle^{5}$ la buona fede in senso oggettivo ha tutt'altro contenuto ed ambito di incidenza. Di essa manca una definizione nell'attuale Codice Civile italiano e il riferimento più prossimo è l'art. 1175 c.c. nel quale si afferma che entrambe le parti del rapporto obbligatorio devono comportarsi secondo le regole della correttezza, il che comporta un costante adeguamento delle principali attività al criterio della buona fede sin dal momento genetico, fino all'esecuzione (art. 1337 c.c.) ${ }^{6}$.

Secondo il disposto derivante dagli articoli menzionati si valuta sia il comportamento in concreto tenuto dai contraenti, ma si va anche oltre nel senso di tener conto delle circostanze, che, ancorché non dipendono dall'attività dei singoli, possano creare un innovativo vantaggio a favore di una delle parti e principalmente del debitore. Di conseguenza nella valutazione della buona fede si ha riferimento non solo al comportamento specifico della parte, ma anche a quello ipotetico che essa avrebbe dovuto tenere per farlo corrispondere a ciò che avrebbe fatto una persona onesta e corretta.

La buona fede oggettiva, pertanto, ha una funzione dinamica che induce a valutare la situazione in sé, anche al di là di quanto gli agenti abbiano fatto. Questa valutazione è così penetrante da consentire di andare oltre l'eventuale rispetto formale della norma?.

In tale situazione è stato osservato che l'espressione buona fede oggettiva, allora, "può essere usata per designare quell'insieme di prestazioni, di diritti, di doveri che, sul piano del contenuto del contratto e dell'esecuzione dell'obbligazione, incombono sulle parti, in base al generico dovere di comportarsi secondo correttezza e, quindi in buona fede» ${ }^{8}$.

Quanto affermato in questa premessa mi spinge ad interrogarmi sulle origini storiche dell'istituto della buona fede oggettiva, chiedendomi in particolare se in realtà già all'epoca del diritto romano esistesse una distinzine tra buona fede soggettiva ed oggettiva e soprattutto sul ruolo svolto da essa nella concreta realtà applica-

5

V. nt. prec

Art. 1337 c.c. - Trattative e responsabilità precontrattuale -. Le parti, nello svolgimento delle trattative e nella formazione del contratto, devono comportarsi secondo buona fede.

7

R. Cardilli, «Bona fides» tra storia e sistema, op. cit., p. $98 \mathrm{~s}$. Si verrebbe a ipotizzare l'applicazione di una norma del caso concreto sostitutiva della dizione esplicitata nel testo di legge, dando vita a un'operazione integrativa del dato normativo che dovrebbe avere il merito di far corrispondere il diritto alla realtà fattuale, ma che ovviamente è molto delicata, e persino pericolosa, potendo essere un potenziale strumento di arbitrarietà. La dottrina più recente è consapevole di queste conseguenze, ma ritiene preferibile correggere ove necessario e sotto l'attenta guida sua e dei Tribunali il dettato di norme che per essere espressione di previsione astratta possono non corrispondere ai criteri di giustizia e di equità ai quali la soluzione dei casi concreti dovrebbe sempre corrispondere.

8

M. Talamanca, La bona fides nei giuristi romani, op. cit., p. 17, la cui osservazione parte dal diritto romano, ma ha valenza generale anche per il diritto contemporaneo. 
tiva, in relazione soprattutto ai rimedi, creati dai giuristi romani per correggere le distorsioni del sistema.

\section{Fides ed exceptio doli generalis}

Nel diritto romano mancava probabilmente una distinzione terminologica tra la buona fede in senso soggettivo ed in senso oggettivo, cui non corrispondeva, invece, incertezza applicativa $a^{9}$. Oltre a ciò, ritengo che sia doveroso premettere che la formazione e la configurazione della categoria della buona fede nel diritto romano si possono cogliere solamente attraverso l'esame dell'inversa e simmetrica elaborazione della nozione di dolo.

Ciò, perché, la buona fede si riferisce a comportamenti opposti a quelli che concretano le ipotesi di dolo e fu costruita, come si dirà, attraverso la protezione fornita nel processo romano contro il dolo.

Una testimonianza del dato apena riportato, così come riferita da autorevole dottrina ${ }^{10}$, si rinviene già nelle XII tavole ${ }^{11}$ : «patronus si clienti fraudem fecerit, sacer esto ${ }^{12}$, nelle quali era imposto al patrono di tenere un comportamento attento e rispettoso, quindi improntato alla fides, altrimenti avrebbe subito la sacertà.

Nella norma non si trova il termine fides, bensì il termine fraus che «esprime il disvalore polarmente opposto al valore fides (il qual valore, come è noto, costituisce il nucleo normativo dell' istituzione della clientela). In questa opposizione polare fides - (fraus) dolus la nozione di fides manifesta la più notevole e duratura fra le costanti della sua evoluzione tecnico giuridica» ${ }^{13}$.

Un esplicito impiego del termine fides, tanto antico quanto quello che si rinviene nella norma delle XII tavole, è testimoniato dal primo trattato tra Roma e Cartagine $^{14}$, in cui le due collettività si impegnavano a rispettare sulla base della demosía pístei (pubblica fede) gli interessi nascenti da negozi di diritto privato, stipulati dal

M. Talamanca, La bona fides nei giuristi romani, op. cit., p. 7 s., il quale afferma (nt. 25): «... i romani non sembrano neppure accorgersi dei diversi significati che concretamente assume il sintagma bona fides (dalla portata apparentemente unitaria) e, dall'altra, lo impiegano nel medesimo contesto nell'uno e nell'altro significato. Ciò potrebbe indurre a ritenere che essi non facessero distinzione fra codeste figure, anche se, probabilmente, il tutto non dava luogo ad incertezze applicative, ma si risolveva nella mancanza di una delimitazione concettuale fra le omonime figure, cui si accompagnava l'assenza di una riflessione sulla natura e sulla funzione di bona fides in senso oggettivo».

10 P. Frezza, Bona fides, in Studi sulla buona fede, Pisa, 1975

118,21 Serv.; ad Aen. 6, 609.

12 (II patrono che inganni il cliente sarà sacro). L'uomo sacro era colui che, poiché dedicato agli dei, poteva essere ucciso da chiunque. Così era appagata la vendetta della divinità.

13 P. Frezza, Bona fides, in Studi sulla buona fede, op. cit., p. 3. La fides, dunque, già nelle più antiche fonti appare come una categoria giuridica che riguarda la condotta di un uomo verso un altro uomo, probabilmente strumento per affermare 'la finalizzazione del diritto alla persona': S. Tafaro, lus hominum causa ius costitutum. Un diritto a misura d'uomo, op. cit., p. 189.

Polib. 3. 22. 9 
mercante cartaginese in zona di influenza romana, e del mercante romano in zona di influenza cartaginese ${ }^{15}$.

Mi pare doveroso evidenziare che nel trattato la fides è sia il fondamento giuridico del trattato stipulato tra le città, sia il fondamento del negozio giuridico stipulato da mercanti appartenenti a collettività diverse. Tanto le due collettività, quanto i due contraenti dovevano rispettare il patto intercorrente tra essi, e astenersi dal compimento di comportamenti dolosi ${ }^{16}$.

In questi due vasti ambiti di applicazione la fides presentava caratteristiche convergenti: la necessità di attendere scrupolosamente ai propri doveri, rispettando gli impegni assunti ed accordando ove ce ne fosse bisogno, protezione a persone bisognevoli ${ }^{17}$.

In questa direzione fu esemplare la disciplina dei contratti, le cui soluzioni ed intuizioni possono fornire utili spunti per il diritto contemporaneo.

In partenza il contratto valeva per ciò che una determinata forma esprimeva secondo il mos maiorum. L'introduzione della fides mutò, invece e completamente la materia, poiché venne introdotta la stipulatio ${ }^{18}$ che sostituì la sponsio ${ }^{19}$ e furono creati i contratti di ius gentium.

I giuristi romani, quindi, intuirono probabilmente che nei contratti «bisognava andare oltre a ciò che era stato detto e previsto, per valutare quello che più confaceva agli interessi in gioco, secondo una visione dinamica ed equilibrata delle rispettive posizioni. [...]. Mi pare di poter affermare che il motivo del passaggio alla fides risiedette nella consapevolezza che la vicenda contrattuale andava oltre ciò che appariva o addirittura si era dichiarato: perché era la regolamentazione di equilibri rispecchianti le aspettative delle persone, le quali risiedevano talora anche in posizioni non espresse, ma che derivavano dall'esperienza del tempo e da istanze di equità

15 P. Frezza, Bona fides, in Studis ulla buona fede, op. cit., p. 3.

16 P. Frezza, Bona fides, in Studi sulla buona fede, op. cit., p. 4: «La presenza della fides come valore normativo proprio dei rapporti internazionali è ampiamente documentata nei testi dei trattati a partire dal sesto secolo a.C. Le parti contraenti solevano affermare in un solenne giuramento la volontà di osservare i patti «fedelmente e senza dolo» [v. i testi nella recente raccolta Staatsverträge des Altertums, vol. II, München, 1962]. Ė evidente che il criterio della responsabilità delle parti contraenti secondo il principio della fides non può essere pensato come esclusivo dei trattati internazionali; era certamente anche il criterio comune della responsabilità nascente dai contratti fra privati; e segnatamente da quei contratti che non obbedivano alle norme positive di una sola città, perché posti in essere fra stranieri».

17 S. Tafaro, Brevi riflessioni su buona fede e contratti, in Collected Papers, XL 3/2006, Novi Sad, 2006, p. 75.

18 La stipulatio era un contratto verbale, concluso attraverso lo scambio formale di domanda e risposta in virtù del quale un soggetto, promissor, si impegnava nei confronti di un altro soggetto, stipulans, che lo interrogava, a compiere una prestazione avente qualsivoglia contenuto; tipica di essa era la conclusione in forma orale e con la pronuncia di parole solenni.

19 La sponsio era la figura tipica di contratto verbale riconosciuta dallo ius civile e antica forma di obbligazione di garanzia. Essa era assunta dallo sponsor rispondendo spondeo alla domanda idem dari spondes? La sponsio poteva accedere solo ad obbligazioni derivanti da stipulatio; era valida anche se era nulla l'obbligazione principale, poiché l'obbligazione assunta dallo sponsor era autonoma rispetto a quella garantita. Era accessibile soltanto ai cittadini romani, durava solo un biennio e non era trasmissibile agli eredi. 
e giustezza, che tendevano a salvaguardare la persona nelle sue relazioni intersog-

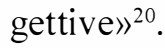

Tuttavia, la fides non poteva tutelare i contraenti da eventuali inganni o sperequazioni, perché anche successivamente all'introduzione del criterio del fit quod dicitur continuava a mantenere un legame stretto tra il negozio giuridico posto in essere e la forma utilizzata per la sua realizzazione.

Fu soltanto la creazione dell'actio de dolo a dare tutela a tutte le situazioni che potessero configurare un approfittamento o un'ingiustizia.

La nozione di dolo fu elaborata dai giuristi romani e articolata in rimedi processuali dall' incessante e progressivo lavorío del praetor, il quale approntò strumenti idonei alla difesa dell'agente contro comportamenti non corretti e nei casi nei quali, pur senza responsabilità di altri, qualcuno si fosse trovato a subíre le conseguenze negative di una situazione non prevista e non voluta nei termini nei quali si era avverata.

Osserviamo piú da vicino quali furono le soluzioni e le tecniche approntate nell'esperienza romana, essenziali per comprendere le proiezioni odierne, per il fatto che quelle nozioni e quelle tecniche si sono diffuse negli ordinamenti contemporanei, avendo particolare incidenza in quasi tutti i sistemi giuridici, con rilevanza decisiva negli ordinamenti ${ }^{21}$ del sistema romanistico del diritto ${ }^{22}$.

S. Tafaro, Buona fede ed equilibrio degli interessi nei contratti, in II ruolo della buona fede oggettiva nell'esperienza giuridica storica e contemporanea, op. cit., p. 570.

La migliore dottrina ritiene che gli ordinamenti giuridici contemporanei differiscono tra loro, non soltanto per la diversità di regole, ma anche per l'ispirazione di fondo della normativa e della caratterizzazione che, in conseguenza di essa, possono assumere; al punto che non di rado princípi e valori di riferimento si riscontrano in tutti gli ordinamenti del sistema. Invero, il fenomeno giuridico è più complesso e non si limita soltanto alle norme; pertanto: "Ogni ordinamento costituisce di fatto un sistema: utilizza un certo vocabolario, che corrisponde a determinati concetti; raggruppa le regole in certe categorie; implica l'uso di certe tecniche per formulare regole, e di certi modi per interpretarle; è legato ad una determinata concezione dell'ordine sociale, che determina il modo di applicazione e la funzione stessa del diritto". Le differenze tra gli ordinamenti, tuttavia, si riducono se si ricorre, a scopo meramente didattico, al concetto di famiglia giuridica, utilizzando il quale è possibile mettere in luce similitudini e differenze. Nel mondo contemporaneo troviamo due principali gruppi di ordinamenti: la famiglia romano-germanica e la famiglia della common-law, che, come è facile immaginare, non costituiscono tutta la realtà del mondo giuridico contemporaneo, ma "accanto alle concezioni che esse rappresentano, o combinandosi con queste concezioni, esistono altri modi di concepire la buona organizzazione della società che continuano ad essere determinanti in un gran numero di società": R. David e C. Jauffret-Spinosi, I grandi sistemi giuridici contemporanei, [cur. R. Sacco], Padova 2004, p. 17 s.

22 L'esistenza e l'individuazione di un sistema romanistico va riscontrata nell'influenza avuta dal dirito romano sulla costruzione degli ordinamenti contemporanei europei ed extra-europei, costituendo una piattaforma comune ma distinta di ordinamenti differenti: v. l'efficace sottolineatura di P. Catalano, Diritto e persone. Studi su origine e attualità del sistema romano, Torino 1990, p. XI, il quale afferma: «La realtà del diritto romano va al di là della sua effettiva applicazione e quindi dei singoli ordinamenti statali. Essa deve essere compresa nella sua complessità temporale e spaziale, dai tempi di Romolo fino al diritto dell'America Latina. A tal fine è utile un uso del termine 'sistema' (tutt'affatto distinto da quello di ordinamento)». I sistemi individuati da Catalano (p. $101 \mathrm{ss}$.) sono quattro «il sistema romanista (di cui quello latinoamericano costituisce un sottosistema), il sistema anglosassone (di cui quello nordamericano costituisce un sottosistema), il sistema socialista ed il sistema musulmano. Non va poi dimenticato che esistono diritti che, per motivi diversi, non possono essere ricondotti ai quattro grandi sistemi mondiali (definiti geograficamente e storicamente): basti qui menzionare il diritto canonico e il diritto indù [...] II sistema romanista è stato strettamente legato, sino al VI secolo d.C. e, in Occidente, ancora oltre, sino al XVIII secolo, all'uso della lingua latina: tale collegamento si è attenuato con le codificazioni moderne: in particolare il 
La recezione e dei concetti e dell'apparato strumentale ad essi conseguente, nell'età contemporanea, ad opera del Code Napoléon ${ }^{23}$, ha ispirato e plasmato le successive codificazioni dell'Europa continentale e dei Paesi ad essa collegantisi.

In questa sede vorrei soffermarmi su alcune implicazioni di rilievo accordate al dolo nell'esperienza romana. Il punto è trattato da parte della dottrina parlando di eccezione generale di dolo (exceptio doli generalis), utilizzando una terminologia che pretende di derivare direttamente dal linguaggio delle fonti ${ }^{24}$.

Essa era un rimedio processuale creato dal pretore verso la fine della repubblica, per paralizzare quelle pretese che, sebbene conformi allo stretto diritto, tuttavia, tenuto conto delle relazioni e affidamenti sorti tra le parti, sarebbero state inique. L'exceptio doli era, probabilmente, frutto di un compromesso fra conservazione giuridica ed esigenze di sviluppo dell'ordinamento. Quando i giuristi rilevavano che una certa soluzione, ispirata a determinati valori emersi a livello di classi dominanti delle quali essi erano espressione, non potesse essere introdotta sul versante dello ius civile attraverso l'interpretatio, mancando un plausibile appiglio per l'operazione interpretativa, poteva ricorrere all'eccezione generale di dolo, ove tale mezzo difensivo in concreto fosse idoneo a raggiungere il risultato voluto.

Ciò posto, ritengo doveroso iniziare l'analisi delle fonti romane sull'argomento in oggetto, partendo da un brano di Ulpiano in cui sembra esserci menzione di dolo:

Code Napoléon (1804) ed il Bürgerliches Gesetzbuch (1900). I codici moderni, elaborati sia in area culturale latina, sia in area culturale germanica, hanno costituito il tramite della diffusione del sistema romanista in aree assai diverse da quella originaria, anche indipendentemente da ogni colonizzazione, bensì, potremmo dire, per rivoluzioni interne: sono i casi, ad esempio, del Giappone e dell'Impero Ottomano (e poi della Repubblica di Turchia)». R. Davide C. Jauffret - Spinosi, I grandi sistemi giuridici contemporanei, op. cit., p. 19 ss. parla di famiglia romano germanica, per evidenziare che "essa si è formata attraverso gli sforzi delle Università europee, che, dal secolo XII, hanno sviluppato, sulla base delle compilazioni dell'Imperatore Giustiniano, una scienza giuridica comune a tutti, adatta alle condizioni del mondo moderno. L'epiteto romano - germanico è scelto per rendere omaggio a questi sforzi comuni, compiuti sia nelle università dei Paesi latini sia di quelli germanici"

23 Il Code Napoléon fu promulgato da Napoleone Bonaparte il 21 marzo 1804. E' il primo Codice in senso moderno ed il modello di tutte le codificazioni successive, effetto diretto della Rivoluzione Francese, nato dall'esigenza di eliminare la molteplicità delle fonti, il particolarismo giuridico e la conseguente incertezza del diritto. L'esportazione e la diffusione del modello, che ha avuto grande fortuna perché è chiaro, semplice, conciso e moderno (ha alla base l'ideologia egualitaria e di libertà), è avvenuta sia attraverso le conquiste napoleoniche sia per la forza dello spirito. Infatti, non si può dimenticare che il termine codice aveva una grande forza evocativa, esso richiamava il Corpus luris Civilis di Giustiniano. La sistematica del Code Napoléon è la seguente: della pubblicazione, degli effetti e dell'applicazione delle leggi in generale più 3 libri: delle persone (art. 7-515); dei beni e delle diverse modificazioni della proprietà (516-710); dei diversi modi in cui si acquista la proprietà (711-2281). Idea centrale del Codice, come si evince dalla sistematica, è la proprietà, il diritto per eccellenza che esprime la conquista della libertà dell'individuo nei confronti dello Stato.

24 In realtà al riguardo ci sono molteplici perplessità, poiché sul piano terminologico sia la distinzione tra dolo speciale e dolo generale sia tra dolus praeteritus e dolus praesens non risultano dalle fonti romane. V.A. Burdese, L'eccezione di dolo generale da Aquilio a Labeone, in Eccezione di dolo generale. Diritto romano e tradizione romanistica, [cur. L. Garofalo], Padova 2006, p. 95; M. Talamanca, La 'bona fides' nei giuristi romani: "Leerformeln» e valori dell'ordinamento, in II ruolo della buona fede oggettiva nell'esperienza giuridica storica e contemporanea. Atti del Convegno internazionale di studi in onore di A. Burdese (Padova-Venezia-Treviso, 14-15-16 giugno 2001), [cur. L. Garofalo], IV, Padova, 2003, p. 34. 
D. 44. 4. 4. 33, Ulp. 1.76 ad ed.: Metus causa exceptionem Cassius non proposuerat, contentus doli exceptione, quae est generalis: sed utilius visum est etiam de metu proponere exceptionem. Etenim distat aliquid doli personam complectitur eius, qui dolo fecit: enimvero metus causa exceptio in rem scripta est 'si in ea re nihil metus causa factum est', ut non inspiciamus, an is qui agit metus causa fecit aliquid, sed an omnino metus causa factum est in hac re a quocumque, non tantum ab eo qui agit, et quamvis de dolo auctoris exceptio non obiacitur, verumtamen hoc iure utimur, ut de metu non tantum ab auctore, verum a quocumque adhibito exceptio obici posasti.

Si è sostenuto che l'aggettivazione generalis presente nel passo vada riferita «a tutt'altro ordine di problemi (l'onnicomprensività dell'exceptio doli, nel caso ancora in relazione all'exceptio metus), che nulla hanno a che fare con la contrapposizione fra dolus generalis e specialis della corrente dottrina romanistica $\rangle^{25}$.

Sarebbero stati i glossatori ad operare la distinzione tra l'exceptio doli generalis e l'exceptio doli specialis, forse dando forma al pensiero che era già presente ai giuristi del Principato ${ }^{26}$.

A dire il vero un altro brano di Ulpiano può ingenerare l'ipotesi che la distinzione della quale ci stiamo occupando potesse essere già stata espressa dai giuristi severiani. Si tratta di un frammento dello stesso contesto, il libro 76 del commento

M. Talamanca, La 'bona fides' nei giuristi romani: «Leerformeln» e valori dell'ordinamento, in ॥ ruolo della buona fede oggettiva nell'esperienza giuridica storica e contemporanea. Atti del Convegno internazionale di studi in onore di A. Burdese (Padova-Venezia-Treviso, 14-15-16 giugno 2001), op. cit., p. 35 nt. 118. In tal senso già A. Burdese, L'eccezione di dolo generale da Aquilio a Labeone, in Eccezione di dolo generale. Diritto romano e tradizione romanistica, op. cit., p. 95. Contra: V. anche V. Mannino, Eccezione di dolo generale e contratti di stretto diritto, in Eccezione di dolo generale. Diritto romano e tradizione romanistica, op. cit., p. 171, il quale afferma: "E' noto che la qualificazione come generale dell'eccezione di dolo si trova chiaramente espressa solo in D. 44. 4. 4. 33, Ulp. I. 76 ad ed., in un contesto del tutto particolare e in contrappunto all'operatività dell'exceptio metus".

26 C.A. Cannata, 'Exceptio doli generalis' e diritti reali, in Eccezione di dolo generale. Diritto romano e tradizione romanistica, cit., p. 235 ss. partendo dalla costatazione secondo la quale nelle fonti romane non compare la denominazione tecnica di exceptio doli generalis, tranne che in D. 44. 4. 4. 33, ricostruisce l'origine di tale qualificazione: "Nella Glossa ordinaria alla lex ora riportata (è ovviamente D. 44. 4. 4. 33) si legge la Glossa seguente: gl. generalis ad D. 44. 4. 4. 33: ut supra I.2 §generaliter. [...].II glossatore, per spiegare il carattere generale dell'exceptio doli, adduceva D. 44. 4. 2. 5 correttamente: egli si riferiva al testo nel suo complesso, e si basava certo sulla sua prima parte, dove si afferma che l'exceptio doli comprende tutte le eccezioni in factum (=non previste espressamente nell'editto): il senso di tale affermazione è infatti che l'attore è sempre in dolo se tiene un comportamento che il pretore potrebbe sanzionare con un'exceptio decretale. Ma siccome per i lettori medievali del Corpus luris le eccezioni in factum sono pure previste nei libri legales, per loro la parte più rilevante del discorso di Ulpiano diventava la seconda, dove egli diceva che l'exceptio doli spetta al convenuto anche contro un attore che non abbia commesso dolo prima di esercitare l'azione, ma sia in dolo nell'esercizio dell'azione: e si tratta precisamente dell'impiego che, dell'exceptio doli, permetteva la parte finale del suo testo edittale, dove viene detto espressamente che mediante l'eccezione viene opposto anche il dolo che si sia concretato con l'esercizio dell'azione stessa. Quest'ultima diventa così la caratteristica specifica dell'impiego dell'exceptio doli in quanto generalis, e ciò ha fatto si che in questo impiego l'eccezione venisse denominata exceptio doli generalis". L'a. a conclusione della sua precisazione terminologica aggiunge: "La terminologia di exceptio doli generalis nel senso di exceptio doli praesentis certo non è romana, ma è stata creata dalla giurisprudenza dell'età intermedia. Possiamo tuttavia nel contempo affermare che la corrispondente nozione era ben presente nel pensiero della giurisprudenza classica, e che l'operazione terminologica e definitoria che partì dalla Glossa rappresentò un correttissimo ed anzi opportuno chiarimento di carattere esegetico di una struttura dommatica romana". 
all'Editto di Ulpiano, al quale apparteneva D. 44. 4. 4. 3. Il giurista diceva che è necessario puntualizzare in modo specifico quale tipologia di eccezione veniva richiesta:

D. 44. 4. 2. 1, Ulp. 1.76 ad ed., Sequitur, ut videamus, in quibus causis locus habeat exceptio et quibus personis obiciatur. Et quidem illud adnotandum est, quod specialiter exprimendum est, de cuius dolo quis quaeratur, non in rem 'si in ea re nihil dolo malo factum est', sed sic 'si in ea re nihil dolo malo actoris factum est: Docere igitur debet is, qui obicit exceptionem, dolo malo actoris factum, nec sufficiet ei ostendere in re esse dolum: aut si alterius dicat dolo factum, eorum personas, specialiter debere enumerare, dummodo hae sint, quarum dolus noceat ${ }^{27}$.

L'uso dell'avverbio specialiter non deve indurre in errore perché sta ad esprimere l'esigenza di indicare in maniera precisa l'autore del dolo lamentato e quindi non ha nulla a che vedere con un eventuale concetto di dolo speciale come configurato dagli interpreti contemporanei.

Anche per altra via l'esistenza nei giuristi romani di espressioni riferite ad una distinzione tra dolo generale e dolo speciale appare infondata. Come si è già detto (rinvia a quale punto) si suppone che il dolo speciale sarebbe potuto essere espresso con l'indicazione di dolus praeteritus, mentre si sarebbe fatto riferimento al dolo generale parlando di dolus praesens.

Al riguardo viene menzionato un altro punto del commento di Ulpiano all'eccezione di dolo:

D. 44. 4. 2. 1, Ulp. 1.76 ad ed.: «Sequitur, ut videamus, in quibus causis locum habeat exceptio et quibus personis obiacitur. Et quidem illud adnotandum est, quod specialiter exprimendum est, de cuius dolo quis queratur, non in rem "si in ea re nihil dolo malo factum est", sed sic "si in ea re nihil dolo malo auctoris factum est". Docere igitur debet is, qui obicit exceptionem, dolo malo auctoris factum, nec sufficiet ei ostendere in re esse dolum: aut si alterius dicat dolo factum, eorum personas specialiter debebit enumerare, dummodo hae sint, quarum dolus noceat».

Quanto alla diversa terminologia di dolus praeteritus in contrapposto a dolus praesens si deve notare che nelle fonti si ritrova soltanto:

D. 44. 4. 4. 18, Ulp. 1. 76 ad ed.: Quaesitum est, an de procuratoris dolo, qui ad agendum tantum datus est, excipit posasti. Et puto recte defendi, si quidem in rem suam procurator datus sit, etiam de praeterito eius dolo, hoc est si ante acceptum iudicium dolo quid facerit, esse excipiendum, si vero non in rem suam, dolum praesentem in exceptionem conferendum. Si autem is procurator sit, cui omnium rerum administratio concessa est, tunc de omni dolo eius excipi posse Neratius scribit. 
Il brano così come è nella dizione pervenutaci non sembra probante riguardo all'esistenza di una distinzione classificatoria. In realtà si tratta di un caso particolare, nel quale: «il dolus praeteritus del procurator stesso rileva soltanto in quanto sia stato nominato in rem suam, ma - a differenza di quanto non avvenga nella terminologia corrente in letteratura - il dolus praeteritus non è il dolo negoziale, ma un comportamento scorretto imputabile al procurator (in rem suam) che - riflettendosi sul presente - rende a sua volta scorretta la proposizione dell'azione, proprio perché il procurator agisce nel suo esclusivo interesse: si arriva così alla conclusione che, l'unica volta in cui si riscontra la contrapposizione con il dolus praesens, il dolus praeteritus non è, in sostanza, se non un particolare caso del dolus praesens stesso in cui l'intende la corrente dottrina ${ }^{28}$.

In conclusione si può ritenere che nelle fonti ed in particolare da parte di Ulpiano non appare delineato alcun concetto di dolo generale «quale dolo indipendente da comportamenti effettuati ante litem contestatam già di per sé considerati dolosi $\gg^{29}$.

E', invece, evidente che pur in assenza di siffatta distinzione, attraverso l'opportuna modulazione dell'eccezione di dolo si riuscì a tutelare la parte debole contro il comportamento non corretto nel quadro di una sostanziale equivalenza «tra le formulazioni dei mezzi giudiziari in cui ci si riferisce alla fides bona ed al dolus malus $»^{30}$.

Ciò perché «i prudentes non richiedevano come essenziale presupposto dell'impiego di tale mezzo difensivo, una intenzione fraudolenta o malevola dell'attore, un inganno o una volontà diretta a pregiudicare l'altrui sfera economica e giuridica, meritevole di particolare riprovazione morale ${ }^{31}$.

\section{Conclusioni}

L'attenzione alle forme di protezione contro il dolus ha spostato l'esame dal piano sostanziale a quello processuale.

Questo è essenziale per le visioni dei romani, i quali non partivano dal riconoscimento dei diritti, bensí dalla tutela giudiziaria (dall'actio), affermando l'esistenza di un diritto solo là dove ci fosse una forma di protezione processuale.

\footnotetext{
28 M. Talamanca, La 'bona fides' nei giuristi romani: «Leerformeln» e valori dell'ordinamento, in II ruolo della buona fede oggettiva nell'esperienza giuridica storica e contemporanea. Atti del Convegno internazionale di studi in onore di A. Burdese (Padova-Venezia-Treviso, 14-15-16 giugno 2001), op. cit., p. $35 \mathrm{nt}$. 118. 29 A. Burdese, L'eccezione di dolo generale da Aquilio a Labeone, in Eccezione di dolo generale. Diritto romano e

30 G. Finazzi, L"exceptio doli generalis' nel diritto ereditario romano, op. cit., p. $250 \mathrm{~s}$.

31 G. Finazzi, op. cit., p. 250 nt. prec.
} 
Il che non esclude che i giuristi romani elaborassero princípi e/o regole, come nel caso del dolo ${ }^{32}$.

Riguardo ad esso furono enunciate soluzioni improntate a princípi, spesso racchiusi in definitiones dai contorni aperti e non tassativi.

Cosí come aperto era l'intero sistema, nel quale fu possibile accordare protezione contro comportamenti colpevoli di un agente (dolus praetritus) o perché la situazione che si era realizzata in un rapporto appariva iniqua o inopportuna (dolus praesens), ma ciò avvenne in base al riconoscimento di valori preordinati, che nel caso erano quelli articolati intorno alla fides.

La duttilità con la quale procedettero sia i giuristi sia i magistrati giusdicenti (pretores in Roma, i governatori nelle 'province') era propria del sistema 'aperto' e risultò esemplare, costituendo un modello efficace di protezione dell'uomo e di perseguimento della giustizia sostanziale.

Per queste caratteristiche la dottrina contemporanea, ivi compresi (ma impropriamente) alcuni romanisti, di fronte alla crisi delle certezze e delle fonti del diritto hanno ritenuto opportuno annodarsi alle soluzioni elaborate nel diritto romano per affermare forme di protezione dell'uomo e dei suoi interessi meritevoli di tutela, pur nei cambiamenti della società contemporanea e di fronte a negoziazioni che non possono essere disciplinate nell'ámbito dei confini degli stati nazionali.

Si è ipotizzata l'esistenza di una categoria generale di dolo derivante dalla compromissione dei doveri di giustezza e correttezza, elaborati all'interno della categoria della fides e si è invocata l'esistenza, la disciplina e l'applicazione, con adeguati strumenti processuali, della 'buona fede oggettiva'. Partendo dalle fonti romane essa è stata ravvisata nei casi nei quali un agente si venga a trovare in condizione di svantaggio obiettivo, pur se non a causa del comportamento di altro agente.

In tale direzione il cammino intrapreso, soprattutto della 'civilistica' è stato poi spedito ed incisivo.

32 F. Schulz, I principi del diritto romano, Firenze, 1995, p. 1 s.: "Questi principi non sono tali da potersi comodamente leggere nei nostri testi. I romani non li esprimono, perché in genere non è nelle loro abitudini l'introspezione né la posizione dei principi; per molti di essi si può dire con certezza che i romani non ne hanno avuto coscienza neppure una volta. É tuttavia non è da pensare che si tratti di fantasie. I principii costruttivi dei giuristi romani devono essere necessariamente riconoscibili nel risultat della loro fatica, e si possono indurre dai singoli fatti della storia giuridica. Anzi, mentre centinaia di particolari dell'ordinamento giuridico romano e della sua storia sono e rimangono necessariamente problematici per lo stato frammentario ed interpolato dei nostri testi, per il riconoscimento delle idee fondamentali e della maniera di pensare di quelli che hanno creato l'ordinamento romano le fonti scorrono abbondanti e limpide. In confronto dei singoli fatti della storia del diritto, i principii nel senso indicato si presentano come una costante; ma naturalmente seguono anch'essi una legge dell sviluppo, e anch'essi hanno una loro storia». 


\section{BREVI RIFLESSIONI SULL'ORIGINE DELL'ECCEZIONE GENERALE DI DOLO}

I giuristi e i magistrati giusdicenti romani elaborarono soluzioni giuridiche duttili attraverso le quali riuscirono a perseguire sia la tutela dell'uomo sia l'attuazione della giustizia sociale.

Partendo dalle potenzialità della fides e della inversa e simmetrica nozione di dolus, i giuristi romani crearono l'exceptio doli generalis, rimedio processuale elaborato dal pretore verso la fine della repubblica, per paralizzare quelle pretese che, sebbene conformi allo stretto diritto, risultavano inique, tenuto conto delle relazioni e affidamenti tra le parti.

Il rimedio processuale, volto ad evitare immotivati abusi conseguenti ad una rigida applicazione del diritto, sorse probabilmente successivamente all'emersione di nuove esigenze e nuovi valori, i quali non potendo essere introdotti sul versante dello ius civile penetrarono nel sistema attraverso l'interpretatio.

Dallo studio delle fonti romane ha preso, poi, avvio la riflessione della dottrina civilistica contemporanea per affermare adeguate forme di protezione dell'uomo e dei suoi interessi, pur essendo consapevole della diversità del contesto storico attuale, caratterizzato dalla crisi della certezza e delle fonti del diritto, anche in conseguenza dei processi di globalizzazione. I giuristi, infatti, l'esigenza di elaborare soluzioni giuridiche adeguate alla società industriale di produzione di massa che allo stesso tempo siano in grado di tutelare l'uomo. Ciò non significa abbandonare i Codici e rinunciare ad applicare i concetti giuridici tradizionali, ma oltre ad essi assume un ruolo centrale l'attività interpretativa, con la quale si compie un'attività di mediazione tra le disposizioni e le esigenze del tempo presente. A tal fine, devono essere messi a disposizione dei giuristi strumenti duttili, così come è la buona fede oggettiva. 


\section{KRÓTKA REFLEKSJA NA TEMAT POCHODZENIA ZARZUTU PODSTĘPU}

Rzymscy juryści oraz sędziowie orzekający o prawie opracowali elastyczne rozwiązania prawne za pomocą którego potrafili zarówno dążyć do ochrony człowieka jak i do sprawiedliwości społecznej.

Wychodząc od potencjału fides oraz odwrotnego i symetrycznego pojęcia dolus, rzymscy prawnicy stworzyli exceptio doli generalis, środek procesowy opracowany przez pretora pod koniec republiki w celu sparaliżowania tych żądań, które, chociaż ściśle zgodne z prawem, okazywały się niesprawiedliwe, po przeanalizowaniu stosunków i zaufania między stronami.

Środek procesowy, stworzony w celu unikania nieuzasadnionych nadużyć wynikających z sztywnego stosowania prawa, powstał prawdopodobnie po pojawieniu się nowych wymagań i nowych wartości, które nie mogły być wprowadzone do ius civile, ale przeniknęły do systemu poprzez interpretatio.

Badania nad źródłami rzymskimi dały impuls rozwojowi myśli współczesnej doktryny cywilnej, która potwierdza odpowiednie formy ochrony człowieka i jego interesów, pomimo świadomości odmienności aktualnego kontekstu historycznego, nacechowanego kryzysem niepewności i kryzysem źródeł prawa, który nastąpił również w wyniku procesów globalizacji. Juryści właśnie potrzebują stworzyć rozwiązania prawne odpowiednie dla społeczeństwa masowej produkcji, które są w stanie w tym samym czasie chronić człowieka.

Nie oznacza to, że należy porzucić kodeksy i zrezygnować ze stosowania tradycyjnych koncepcji prawnych, ale poza nimi nabiera głównej roli działalność interpretacyjna, która dokonuje się poprzez mediację pomiędzy rozporządzeniami prawnymi a wymaganiami obecnych czasów. W tym celu muszą być oddane do dyspozycji prawnikom elastyczne środki tak jak i dobra wiara. 


\section{BRIEF REFLECTION ABOUT THE ORIGIN OF THE PLEA OF DECEIT}

Roman jurists and judges deciding about the law, developed a flexible legal arrangement that enabled them to seek to protect both the human being and social justice.

Starting from the potential of fides and the inverse and symmetric concept of dolus, Roman lawyers created the exceptio doli generalis, a procedural measure developed by the praetor at the end of the Republic in order to petrify these requests, which, though strictly in accordance with the law, proved to be unfair after examination of the relationship and trust between the parties.

The procedural measure was created in order to avoid unfounded abuses resulting from rigid application of the law. It was probably after the appearance of new demands and new values, which could not be introduced to the ius civile, but which penetrated into the system through interpretatio.

Research on the sources of Roman law, gave impetus to the development of modern civil doctrine, which confirms the appropriate forms of protection for a man and his interests, despite awareness of the differences of actual historical context marked by a crisis of uncertainty and crisis of sources of law, which took place as a consequence of the process of globalization. These are the jurists who need to create legal regulations appropriate for a society of mass production and which at the same time are able to protect human beings.

This does not mean that one should abandon the codes and refrain from the use of traditional legal concepts, but besides them, the interpretative activity - which is carried out through mediation between the regulations and legal requirements of present times, - should gain the main role. That is why lawyers have to be given flexible measures and also good faith.

Key words: deceit, application of law, interpretation, Roman law 